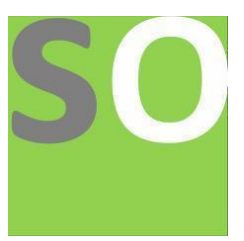

Article title: A Survey on The Impact of Early Speech and Vocal Changes on Young Onset Parkinson's Disease. Authors: Stephanie Liangos[1]

Affiliations: Escuela de Formación Superior, SAERA, Spain[1]

Orcid ids: 0000-0002-8639-1190[1]

Contact e-mail: stefieliangos@gmail.com

License information: This work has been published open access under Creative Commons Attribution License http://creativecommons.org/licenses/by/4.0/, which permits unrestricted use, distribution, and reproduction in any medium, provided the original work is properly cited. Conditions, terms of use and publishing policy can be found at https://www.scienceopen.com/.

Preprint statement: This article is a preprint and has not been peer-reviewed, under consideration and submitted to ScienceOpen Preprints for open peer review.

DOI: 10.14293/S2199-1006.1.SOR-.PPALOS3.v1

Preprint first posted online: 05 November 2021

Keywords: young onset parkinson's disease, hypokinetic dysarthria, speech, voice, psychosocial impact 


\title{
A Survey on The Impact of Early Speech and Vocal Changes on Young Onset Parkinson's Disease.
}

\section{Author: Stephanie Liangos}

\begin{abstract}
Patients with Young Onset Parkinson's Disease may have a long medical journey to receive their diagnosis due to their atypical age. Parkinson's Disease is typically diagnosed in a geriatric population and thus assumed to be a late-onset neurodegenerative disorder. Therefore, when younger people approach clinicians with parkinsonian symptoms, they are typically overlooked as they do not meet the age criteria, and thus the diagnosis may be missed or delayed. In late-onset Parkinson's Disease, a classic primary symptom pertains to voice and speech disorders due to the high prevalence of hypokinetic dysarthria. Thus, a review of speech and voice deficits that are seen prior to or within the time frame of diagnosis can highlight the speech and vocal patterns clinicians may see within a younger population. This could provide an effective tool for clinicians to make a quicker diagnosis for patients and administer medication such as Levodopa without having the patient go through rigorous, time-consuming testing. Furthermore, within neuroscience, little attention is paid to the impact of early speech and vocal changes. Therefore, this study would also like to explore the impact of these changes, highlighting the urge for clinicians not to stigmatise younger patients by age to receive a rapid diagnosis and treatment. This study follows the proceedings of a survey methodology via a formulated questionnaire
\end{abstract}


inserted in a Google Form containing 12 statements, which contained closed-ended questions (Yes/No indicators) and open-ended questions where the participants indicated their answer by filling in a short statement regarding their experience. The statements contained questions about the diagnosis of Parkinson's Disease, the speech and vocal changes experienced, the socio-social effects of the speech and vocal changes on their personal lives and if they found that medication helped their vocal and speech symptoms. The questionnaire yielded a total of 43 participants with young-onset Parkinson's Disease. The results indicated that most of the participants suffered from speech and vocal changes, which resembled the clinical profile of severe hypokinetic dysarthria, typically seen in later stages of Parkinson's Disease in late-onset. In addition, the changes in speech and voice were so impactful that they caused significant distress in the psychosocial domain of their lives. Despite the severity of the speech and vocal changes, most participants struggled to receive a diagnosis, while hardly any received appropriate speech therapy treatment to aid their overall quality of life. Thus, this study concludes that the results of this study are essential to break stigmas and open the conversation in neuroscience and neurology on YOPD. Improvement clinical knowledge of this unique subtype of Parkinson's needs to be stressed amongst clinical practitioners that age of onset does not play a role in managing, treating, and diagnosing Parkinson's Disease.

Keywords: young onset parkinson's disease, hypokinetic dysarthria, speech, voice, psychosocial impact 
Table of Contents

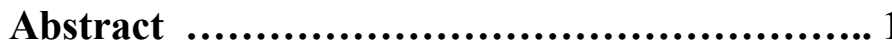

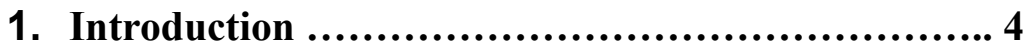

1.1. Parkinson's Disease ........................................ 6

1.2 Age and Categorisation of Onset in Parkinson's Disease ................... 6

1.3 Motor and Non-Motor Symptoms ........................................... 7

1.4 Speech and Voice Changes in Parkinson's Disease .................... 7

1.5 Diagnosing Parkinson's Disease ..........................................9

2. Objective and Research Questions ....................................... 11

3. Methods and Material ........................................13

3.1 Questionnaire ........................................... 13

3.2 Informed Consent............................................. 14

4. Results .........................15

4.1 Participant Demographics ........................................ 15

4.2 Analysis on Self-Reported Speech and Vocal Changes Reported in the Survey (............................. 16

4.2.1 Speech Changes ......................... 16

4.2.2 Vocal Changes ........................... 17

4.2.3 Impact of Vocal and Speech Changes 19

5. Discussion

\section{1}

6. Limitations ......................... 28

7. Proposal for Future Research 29

8. Conclusion ........................... 30

Acknowledgements ......................... 31

Conflict of Interest ............................ 31

Reference List.......................... 32

Appendices ........................... 37

Table of Figures

Figure 1. Initial Symptoms Self-Reported by participants............................................ 16

Figure 2. Word cloud with the most frequent mentions of vocal and speech changes.............................................. 18 


\section{Introduction}

"I became conscious that my voice tended to sound flat and lacking in expression...My voice had become softer, and I was unable to enunciate certain words clearly...If I went on talking, my voice would fail, and I could do no more than whisper"

(Thompson, 1995, p.323)

Neurodegenerative disease is the term given to the disorders that, according to Przedborksi (2003), are primarily age-dependent, causing progressive degeneration of the central nervous system. The following may serve as a brief overview on standard clinical features for neurodegenerative diseases:

i) All neurodegenerative diseases affect a specific subset of neurons.

ii) Are relentlessly progressive until death, without a clear explanation and could either be inherited or acquired.

iii) Although neurodegenerative diseases are often age-related, they can occur as juvenile diseases, but this is relatively rare.

iv) Neurodegenerative diseases present are neuronal cell death, the disappearance of cell bodies and glial proliferation.

v) The degeneration of neurons is not reversible, and although pharmaceuticals may give marginal or temporary improvement to the disease there is no cure to reverse the effects of the degeneration. 
vi) Cognitive impairments and dementia are common manifestations of neurodegenerative disorders but are not seen in all forms (Przedborski et al., 2003).

Specific disorders that can occur due to neuronal degeneration are Huntington's Disease, Amyotrophic Lateral Sclerosis, Multiple Sclerosis, Alzheimer Disease and finally, the disease that pertains to the topic of this study, Parkinson's Disease (PD hereafter).

\subsection{Parkinson's Disease}

PD is a complex neurodegenerative disease that was first described as "shaky palsy" by James Parkinson (1817), and then further coined as Parkinson's Disease by Jean-Martin Charcot (Parkinson, 2002). PD is the second most common neurodegenerative disease after Alzheimer's Disease, and it affects $1 \%$ of the population above 60 years of age.

The disease manifests as a consequence of the progressive loss of dopaminergic neurons in the substantia nigra compacta of the midbrain causing motor and non-motor symptoms to manifest (De Lau et al., 2005). The causation of PD is widespread and indications show that it can be due to multiple factors, which can be familiar and present as idiopathic and rare, trauma, age or even environmental factors such as pesticides (Duffy, 2012). Out of all the neurodegenerative diseases, Parkinson's is the only one which has specific medications that are tailored to this given disease. The discovery of the non-dopaminergic deficiency has led to the development of several medications, most notably Levodopa which although doesn't stop the deterioration of the dopaminergic neurons, it does aid patients with a significant improvement in 
their quality of life. Patients who take Levodopa see a difference in the primary symptoms of Parkinson's as the medication helps target motor symptoms become less pronounced and regulates atypical motor movements. However, as the disease progresses, the Levodopa dosage will increase which may cause the patient to have nausea, hypertension, confusion, stimulant psychosis, auditory and visual hallucinations.

\subsection{Age and Categorisation of Onset in Parkinson's Disease}

Although the mean age of diagnosis for PD is 60 years of age, it has been reported that even from the age of 20, symptoms of Parkinson's are present -however rarely reported- or they are dismissed (De Lau et al., 2005). Although PD is recognized as an idiopathic disease, with respect to age of onset the categorization has been subdivided into early (YOPD; young-onset PD) whose age is 50 years and younger, and late (LOPD; Late/regular onset PD) whose age is 50 years or older (Fénelon et al., 2000; De Lau et al., 2005). It's interesting to note, although often PD is regarded as a rare neurodegenerative disorder that mainly pertains to to a geriatric population, reported cases of YOPD is becoming much more frequent and even going into juvenile age groups, with the youngest person to have been diagnosed with Parkinson's Disease being twelve years of age (Anwar et al., 2019). YOPD patients are considered to have typical PD due to resemblance to LOPD patients in both clinical and pathological features. However, despite the similarities between YOPD and LOPD, clinicians lack consideration or strong reluctance towards diagnosing younger patients with PD, missing subtle symptoms which may lead to significant delays in diagnosis. This may also result in extensive use of medical resources, with 
an increased number of medical investigations and visits to the same or multiple neurologists prior to a final diagnosis which may take many years.

\subsection{Motor and Non-Motor Symptoms}

The motor symptoms that occur as a clinical characteristic in PD include resting tremor, rigidity, bradykinesia, hypokinesia, loss of postural reflexes, masked facial expressions and writing disorders such as micrographia. However, perhaps the most prominent motor deficit that according to previous researchers (Ho et al., 1999; Duffy, 2012), which occurs in 90\% of PD patients pertaining to distinctive motor speech disorders which are collectively referred to as a Hypokinetic Dysarthria (Darley et al., 1969; Poluha,1998; Yunusova et al., 2008; Brabenec et al., 2017). Furthermore, as degeneration occurs, other areas of the brain that are not associated with the Basal Ganglia may be affected causing non-motor symptoms as well as motor symptoms in Parkinson's Disease. The non-motor symptoms are broad, however, some of the most commonly reported non-motor symptoms are fatigue, joint pain, depression, gastroenterological issues, restless leg syndrome, mild memory and thinking issues, lack of saliva control and swallowing issues (Serván, 2018 )

\subsection{Speech and Voice Changes in Parkinson's Disease}

Hypokinetic Dysarthria (HD hereafter) is a distinct motor speech disorder that is associated with basal ganglia control circuit pathology (Duffy, 2012). HD may affect any or all 
parts of the respiratory, phonatory, reasonatory, and articulatory levels of speech. However, it is most observant in voice, articulation, and prosody. As it is "hypo", the disorder causes reduced movement in the muscular control of the larynx, planning and programming of the articulators and oralkinesia (Kegl et al. 1999). Common deviant speech dimensions that are encountered in HD is described as monopitch, mono loudness, speech rate disturbances, reduced stress, palilalia, low pitch, harsh and breathy voice quality, inappropriate silence, short rushes of speech, and increased acoustic noise (Darley et al., 1969). Affected patients with HD often describe effects that may provide clues for a PD diagnosis such as they are frequently told their voice has suddenly become a lot quieter or weak (Duffy, 2012). Furthermore, some report that the rate of speech becomes very fast and as a result speech cluttering occurs (Duffy, 2012). Additionally, some complain they often find it hard to initiate conversation and will often repeat phonemes, words or sentences, as they will be contaminated by stuttering. Some report the upper lip starts to feel stiff (Duffy, 2012). It has been reported that initially a patient suffering with PD may minimize or completely deny the changes that have occurred in their speech, whilst others around them will be persistent of the changes that have become highly noticeable (Duffy, 2012). As a consequence, by ignoring the speech deficits, they prolong becoming diagnosed with PD. Often a diagnosis of PD happens much later in life than when the initial symptoms started to appear. By this point, in the patient's life their PD will have progressed as we enter the more advanced stages of the HD in which the most conventional speech and voice deficits manifest such as a metallic quality to the voice, mumbling, reduced loudness, reduced utterance of sentences, rapid or galloping speech (Duffy, 2012). Furthermore, the patient will show masked facial expressions, tremulous jaw, lip and tongue begins. These classic clinical manifestations 
will allow clinicians to make a PD diagnosis as these symptoms are very prominent within the disorder. However, non-conventional speech deficits which may occur in the early stages of the disease, are less clinically interpretable and also less reported (Mekyska et al. 2016). Although there is a preconceived opinion happen at later stages in PD, in self reported studies, measures indicated that between $40 \%-70 \%$ of early onset PD patients have significant speech and vocal changes even prior to receiving a diagnosis (Volont et al., 2002; Sung et al., 2010; Ciucci \& Hoffman, 2012). Due to the late diagnosis most PD patients receive, clinicians always expect to see the most severe HD symptoms in Parkinson's disease as mild HD symptoms although can occur very in early in the course of the disease, and even in the prodromal stages of the disease, the manifestation of the initial stages of the speech and vocal changes are often not reported (Skodda, 2012; Duffy, 2012). Thus, knowing the initial speech and vocal changes can be a useful tool in identifying an early PD, so patients can receive pharmaceutical treatments, early speech, and occupational therapy, which although will not cure the disease, it will provide patients with a better quality of life and preparation against the effects of PD which can overall lead to a more favorable prognosis for PD patients.

\subsection{Diagnosing Parkinson's Disease}

The process of being diagnosed with Parkinson's may be long, and patients typically have to wait for a set of examinations to be done with multiple clinicians which is time-consuming. As time is a factor against patients with neurodegenerative diseases, it is of empirical importance that patients suspected of neurodegenerative diseases be diagnosed promptly. Given the early 
deficit reported in speech and voice in PD, if clinicians suspect a young-onset PD knowing the early speech and vocal markers, they can refer patients immediately to speech therapists for an assessment of speech. This option would be much more accessible and faster to obtain than other tests a patient with suspected Parkinson's disease may need, such as an MRI, which may have limited availability in some countries (Illes,1989). Thus, as the primary deficit of Parkinson's is seen in speech and vocal patterns through early stages of HD, a review on the early deficits and changes seen in speech and vocal patterns could provide an effective tool for clinicians to make a quicker diagnosis for patients. Ultimately this means a quicker administration of medication such as Levodopa and related therapies without having the patient go through a rigorous time-consuming testing procedure, and thus a key motivation to conduct this study. 


\section{Objective and Research Questions}

The aim of this research is to understand what are the early speech and vocal changes that have been self-reported by patients with young onset Parkinson's Disease (YOPD) and whether these speech and vocal changes are significant enough to have an impact on their day to day living. The results of this study could aid in creating future assessment tools to come to a quicker diagnosis for PD in patients that are clinically young for the disease (less than 50 years of age). Furthermore, this research seeks to shed light on YOPD and break preconceived stereotypes that PD is a neurodegenerative disease that we can expect to see within a geatric population exclusively. The literature that was addressed in the theoretical framework on PD suggested that mean age of a diagnosis for PD is 60 years of age, and at this stage of the diagnosis, the staging of PD has usually progressed sufficiently, the patient is presenting with classical PD signs such as tremor, bradykinesia, postural insufficiency, signs of hypokinetic dysarthria which will prompt the individual to visit a neurologist. Given the older age of the individual, it is most likely the neurologist will conclude the individual has PD promptly. However, PD can present symptoms in the preclinical stages much earlier than the mean age of diagnosis which would be considered young on-set PD (Grant et al., 2013). If these subtle signs are missed and clinicians find it difficult to come to PD diagnosis based on a young age, there is a window of opportunity for an early intervention that is lost and decreases the quality of life of the patient who is being misdiagnosed (Grant et al., 2013). Thus, what often occurs is that young onset PD is overlooked not only in clinical practice but also in research. Currently there is no research within the literature that specifically focuses on YOPD in regards to speech and vocal changes. Thus, there 
is a gap in research which this study aims to open. The research questions in this study will take on a three-fold approach and are as follows:

R1. What are the early speech and vocal changes that have been self-reported by Young-Onset Parkinson's Disease Patients?

R2 What is the impact on the participants of this study as a consequence of self-reported speech and vocal changes by Young-Onset Parkinson's Patients?

R3. Are the speech and vocal changes reported significant enough in order to assist a quicker diagnosis in Young-Onset Parkinson's Disease? 


\section{Methods and Material}

This study follows the proceedings of a survey methodology via a formulated questionnaire inserted in a Google Form. As there is scarce literature on YOPD patients, this questionnaire will aid in constructing a demographic profile that will depict the speech and vocal characteristics within the sample of YOPD participants that are being surveyed. The questionnaire was distributed in private YOPD communities via groups found on online social media platforms such as Facebook and Instagram. Furthermore, boolean keywords in the form of hashtags were used on instagram in order to find individuals with YOPD who have used \#YOPD \#youngonsetparkinsons on their social media profiles that may have been willing to partake in this research study. A minimum of thirty participants was set in order for the study to be conducted.

\subsection{Questionnaire}

The questionnaire was designed via google forms containing 12 statements, which contained closed-ended questions (Yes/No indicators) and open-ended questions where the participants indicated their answer by filling in a short statement regarding their experience. The statements contained questions about the diagnosis of Parkinson's Disease, the speech and vocal changes experienced, the socio-social effects of the speech and vocal changes on their personal lives and if they found that medication helped their vocal and speech symptoms. The questions on the questionnaire were carefully formulated to ensure that they can be easily understandable 
in terms of clarity and readability, and adapted to difficulties which one may have with Parkinson's Disease. Furthermore, the timing estimate to complete the questionnaire averaged ten minutes.

If a participant cannot answer a question, they have the option for some of the questions to select the "I do not know" or equivalent "No" answer option. Furthermore, they could also choose to refrain from responding if they felt the question did not apply to their specific circumstance. For instance, if they indicated they had no vocal or speech changes, they did not need to answer the question which pertained to how their speech and vocal changes affect them socially.

\subsection{Informed Consent}

All subjects which participated in this study provided informed consent for the inclusion in the study prior to conducting the questionnaire. An example of informed consent may be seen in Appendix A. Each person included was informed of the objectives and content of the study, their rights and was asked to give their consent on google forms by indicating that they understood the purpose of this study, their rights, and how the information they gave would be utilized. As this is an individual questionnaire, it was not deemed necessary to send the protocol to an ethics committee. 


\section{Results}

The survey yielded a total of 45 respondents; however, four respondents were excluded from participation in the study as their reported age of diagnosis for Parkinson's Disease was above 60 years of age, and thus, did not meet the inclusion criteria on age in order to qualify as being diagnosed with YOPD. Thus, in total, the survey yielded 41 respondents with YOPD.

\subsection{Participant Demographics}

It is important to note that given the informed consent anonymity was promised to the participants, therefore the demographic is limited to the information only that is crucial in order to answer the research questions. Therefore, given the data that was made available, the primary reported gender was female $(\mathrm{n}=25 ; 61.3 \%)$ whilst the remaining were males $(\mathrm{n}=16 ; 39.0 \%)$. Thus, females in this sample with YOPD were double the number of men who had YOPD. The average age for which the participants were diagnosed with YOPD was 41 years and eight months (age range 15-59). However, on average most participants first noticed the initial symptoms of parkinsonism five years prior to their diagnosis at an average age of 35 years (age range from 12-55). Additionally, $14.6 \%(\mathrm{n}=6)$ of the participants reported a familial history of Parkinson's Disease, whilst the remaining $85.4 \%(n=35)$ reported no previous familial history of Parkinson's Disease. In addition, 53.5\% of the participants reported difficulty from their medical team to be diagnosed with YOPD primarily due to their age and the preconceived notion from the medical team that they were "too young" to have Parkinson's Disease. 
The initial symptoms which manifested in which encouraged participants to seek medical attention prior to their diagnosis were most commonly reported as due to tremors $(22.4 \%)$, anxiety (10.3\%), gastroenterological issues $(8.6 \%)$ and changes in gait (6.9\%). A complete overview of the symptoms reported by the participants may be seen below in Figure 1.

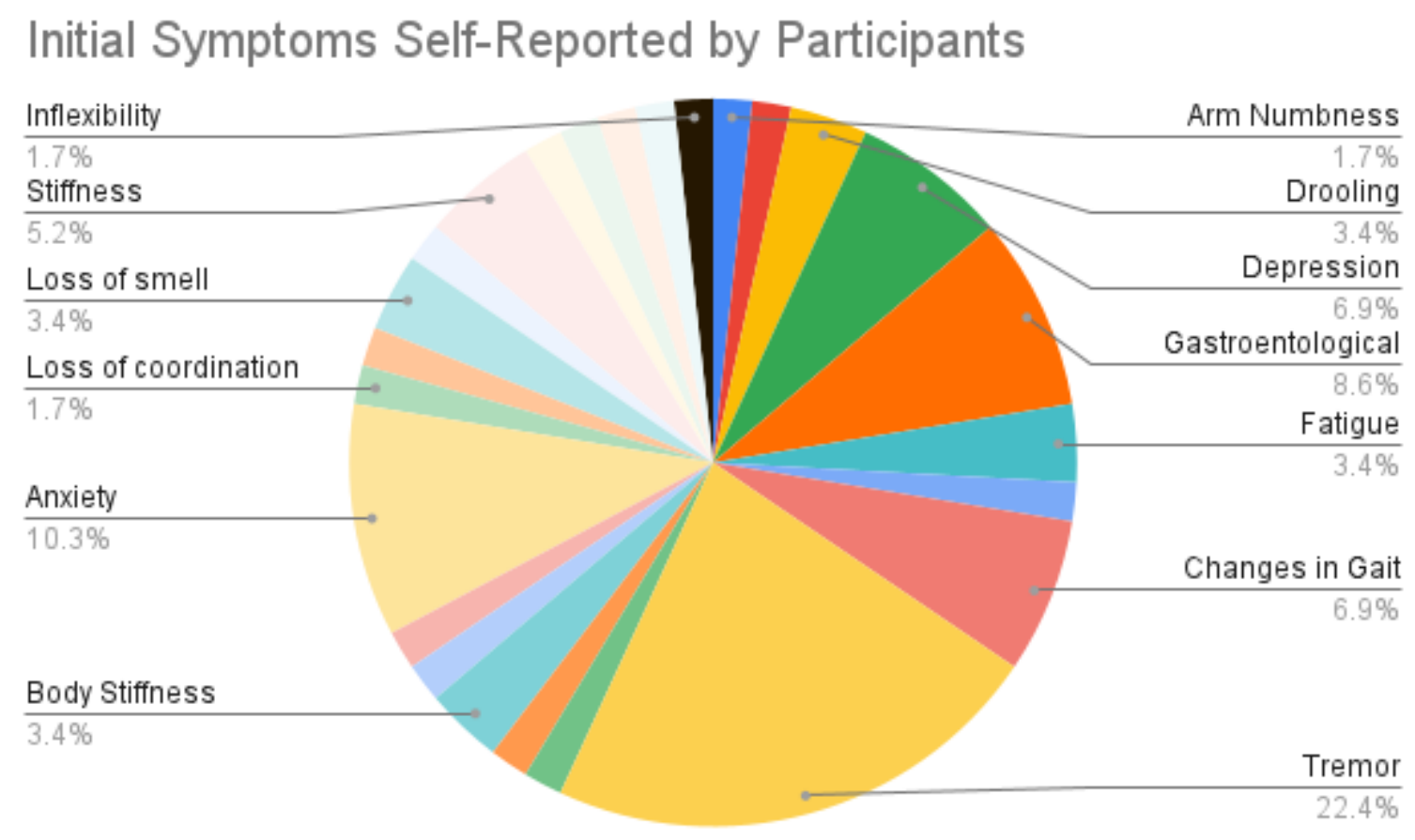

Figure 1. Initial Symptoms Self-Reported by participants.

\subsection{Analysis on Self-Reported Speech and Vocal Changes Reported in the Survey}

\subsubsection{Speech Changes}

Concerning changes in speech, self-reported accounts of speech changes prior to their YOPD diagnosis were made by 12 participants $(12 / 41 ; 29.2 \%)$, whilst the remaining 29 
participants reported no changes in their speech prior to their YOPD diagnosis. The most common reported speech changes were mumbling $(4 / 12 ; 33.3 \%)$, slurred speech $(4 / 12 ; 33.3 \%)$, inappropriate silences $(2 / 12 ; 16.6 \%)$ and inappropriate speech rate $(2 / 12 ; 16.6)$. These reports are consistent with deviant speech markers that pertain to the speech component of prosody in which they are consistent with severe hypokinetic dysarthria (Darley et al.1969). In addition, after receiving their YOPD diagnosis, an additional 5 participants reported changes in their speech which varied to manifest from 3 months to 10 years. The self-reported changes in speech were stuttering $(1 / 5,20 \%)$ slurred speech $(5 / 5 ; 100 \%)$, jargon speech $(1 / 5 ; 20 \%)$, mumbling $(2 / 5$; $40 \%)$ and variable speech rate $(2 / 5 ; 40 \%)$. All symptoms are indicative of a manifestation of severe hypokinetic dysarthria (Darley et al.,1969). Thus, in total, 17 participants $(17 / 28 ; 60.7 \%)$ had experienced speech changes, whilst 12 of them had reported speech changes prior to their formal diagnosis.

\subsubsection{Vocal Changes}

Concerning self-reported vocal changes, 18 participants reported vocal changes prior to their YOPD diagnosis $(18 / 41 ; 43.9 \%)$, whilst the remaining 23 participants reported no changes in their voice prior to their YOPD diagnosis $(23 / 41 ; 56 \%)$. The most common reported vocal changes were monoloudness $(10 / 18 ; 55.5 \%)$ low pitch $(7 / 18 ; 38.8 \%)$ breathy voice $(6 / 18 ; 33.3 \%)$. The vocal reports mentioned by the participants are consistent with deviant vocal markers that pertain to the vocal components of phonatory-prosodic and phonatory-respiratory-prosodic, which are consistent with deviant vocal markers that pertain to positive indicators of severe 
hypokinetic dysarthria (Darley et al.1969). Additionally, after receiving their YOPD diagnosis, an additional 10 participants reported changes in their speech which was first noticed from a range of 3 months to 10 years, as those who reported speech changes after they were diagnosed with YOPD. The reported vocal changes were monoloudness $(4 / 10 ; 40 \%)$, breathy voice $(3 / 10$; $30 \%)$ low pitch $(4 / 10 ; 40 \%)$. All symptoms are indicative of a manifestation of severe hypokinetic dysarthria (Darley et al., 1969).

To sum up, 35/41 (85.3\%) participants reported having already developed speech and vocal changes as a consequence of YOPD. A full overview of the most frequently reported speech and vocal changes may be seen in the word cloud constructed in Figure 2.

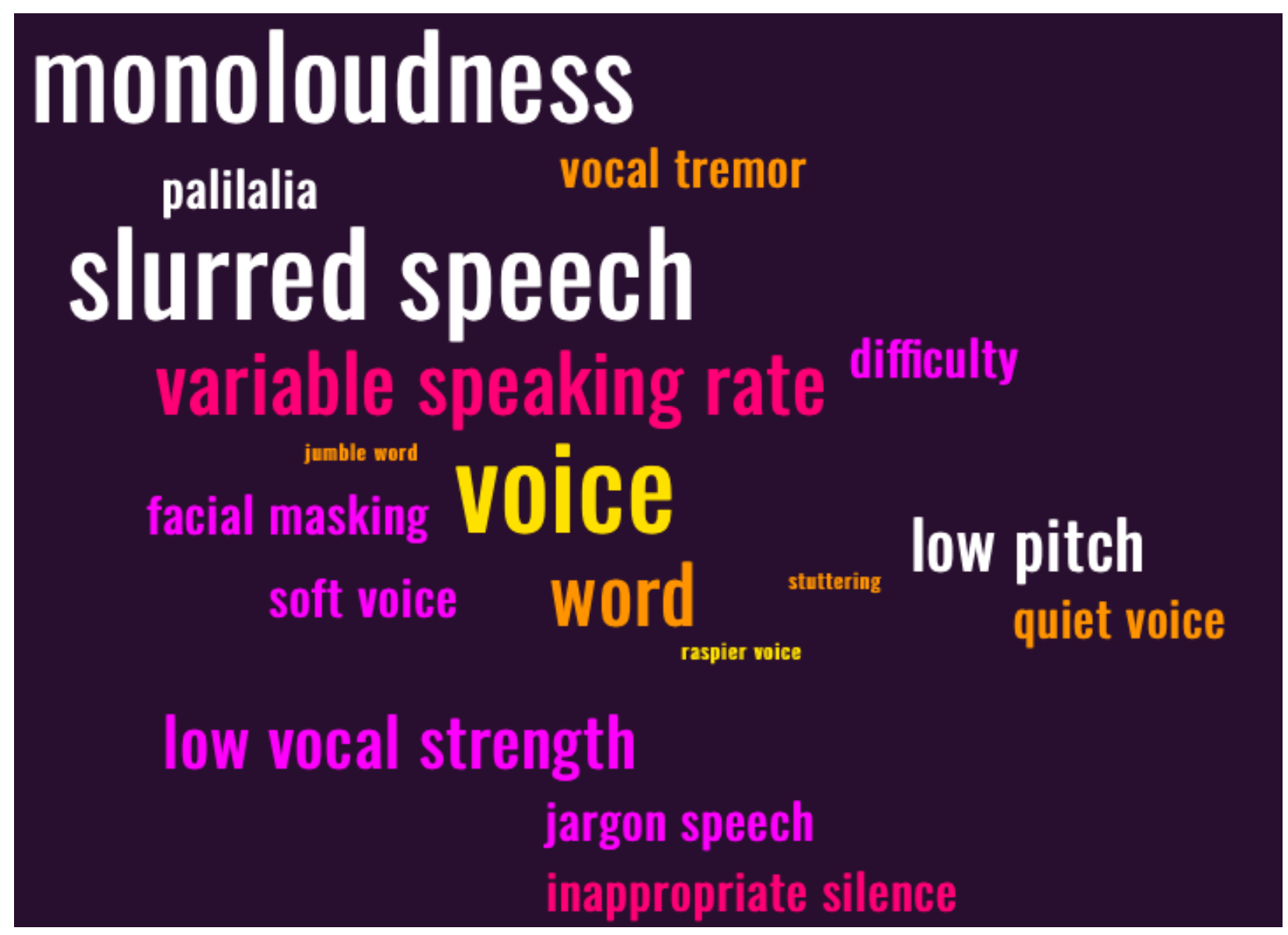

Figure 2. Word cloud with the most frequent mentions of vocal and speech changes. 


\subsubsection{Impact of Vocal and Speech Changes}

Despite the deviant speech and vocal markers mentioned above, which were indicative of prominent characteristics of severe hypokinetic dysarthria, the majority of the participants (79.1\%) had not been referred to a speech therapist by their medical team and despite 31 of these participants $(31 / 35 ; 88.5 \%)$ which reported that their speech and vocal changes significantly impacted their day to day living. Below are extracts of quotes provided from the participants explaining how vocal and speech changes impacted their day to day living:

1. "It can make it hard to communicate and the drooling can put me in a say it don't spray situation"

2. "I continued to work as a teacher/coach after my diagnosis however now I struggle with getting people to understand what I am saying"

3. "Significant impact for my work so I had to stop teaching"

4. "I needed to consciously elevate my voice at all times"

5. "I avoid social situations because people get angry when I stare blankly thinking "I can talk!"

6. "Make talking to others hard. I was fearful and embarrassed"

7. "Talking clearly is exhausting and sometimes I feel like my mind and my mouth are at two different speeds" 
8. "I would have to repeat myself a lot because people didn't hear me. I sometimes had slurred speech and they didn't understand me"

9. "Was difficult/embarrassing in meetings"

10. "Isolated, frustrating repeating myself"

11. "Extremely disturbing and isolated me from others difficult to socialize"

The demographic characteristics showed a high anxiety and depression rate as a symptom to seek professional help before their Parkinson's Diagnosis. From the statements made above, we can see the impact that speech and vocal changes had on participants and how this may lead to feelings of isolation, decreased self-perception and potentially increase social anxiety.

Furthermore, although the administration of the drug Levodopa can help decrease speech and vocal symptoms in Parkinson's Disease, in the sample of this study those who suffered from speech and vocal changes, 11/35 participants (31.4\%) reported that Levodopa helped alleviate the speech and vocal changes.

Finally, many of the participants $(46 \%)$ reported receiving a late or misdiagnosis despite clear indicators in their motor and non-motor symptomatology that they had Parkinson's Disease due to a lack of awareness of YOPD by the practitioner and the preconceived idea that Parkinson's Disease is only seen in a geriatric population. The participants provided the following comments:

1. "Doctors and providers need to be educated about YOPD symptoms"

2. "I knew something was wrong early but I had monsters for doctors and parents" 
3. "I believe that YOPD is actually misdiagnosed and that many of us have had it since we were much younger."

4. "You are never too young to be diagnosed."

Thus, the participants expressed emotions that correlate to feeling stigmatized by their age, and therefore did not receive the treatment necessary at an early enough time, which is crucial in neurodegenerative diseases like Parkinson's Disorder.

\section{Discussion}

The present study investigated the speech and vocal changes that have been self-reported by participants which conducted a survey with Young Onset Parkinson's Disease (YOPD). These participants had to indicate whether the self-reported speech and vocal changes were significant enough to impact their day-to-day living. The results of this study could provide critical findings which can aid clinical practitioners to become more aware of the salient vocal and speech changes in a clinically younger population as opposed to an older population with Parkinson's Disease (PD). This is particularly important as mentioned in the theoretical framework of this paper; PD is connotated with a preconceived stereotype concerning neurodegenerative diseases, which researchers and clinicians regard to occur exclusively within geriatric populations. Generally, patients with PD are susceptible to be stigmatized by their parkinsonian experience which affects their everyday life. This would be amplified for those patients who are considered 
too young to have neurodegenerative diseases and yet still show symptoms (Maffoni et al., 2017). Thus, many with YOPD become susceptible to becoming misdiagnosed and have a late diagnosis in which, during this time; appropriate medication, therapies and interventions to aid their motor and non-motor symptoms will not be provided (Špica et al., 2012). Therefore, this study highlights the overall experience and symptoms which may present in YOPD patients and thus drawing attention in research, neuroscience and clinical management of the awareness of PD in atypically young populations. Thus, with the results outlined in the previous section, the three main research questions will be addressed in the following section.

R1. What are the speech and vocal changes that have been self-reported by Young-Onset Parkinson's Disease Patients?

Regarding R1, an array of speech and vocal changes were reported by the participants of this study. These speech and vocal changes were evident before their diagnosis and/or developed within the time frame of ten years after their initial diagnosis of PD. The speech changes reported from the participants of this study can be categorized into the speech cluster of phonatory-prosodic as significant prosodic insufficiency was reported. The participants in this study described variable speech rates, mumbling, slurred speech, stuttering and jargon speech. As well as the self-reported speech changes, vocal changes were also noted within the participants. The vocal changes that the participants in this study described were monoloudness, low pitch, and breathy voice. The vocal changes mentioned by the participants are consistent 
with deviant vocal markers that pertain to the vocal components of phonatory-prosodic and phonatory-respiratory-prosodic.

The reported speech and vocal changes by the participants of this study reflect the general clinical profile of hypokinetic dysarthria and the prominent deviant speech characteristics (Logemaan et al., 1978). Hypokinetic dysarthria reflects abnormalities at all levels of the speech system, and these abnormalities are associated with phonatory, articulator activities, which cause abnormal prosodic features like the ones mentioned by the participants in the study. Furthermore, the participants' reported speech and vocal changes are often seen in very late stages of PD in which the severity of the speech changes due to hypokinetic dysarthria are very prominent. Patients with hypokinetic dysarthria often complain that their voice is too weak or quiet or their speech is too rapid. Additionally, they may also struggle to start a conversation as they cannot find their voice. Hypokinetic dysarthria will give patients a distinctive flat, attenuated, fused and accelerated speech pattern. Often the speech and vocal patterns of hypokinetic dysarthria are characterized by monopitch, monoloudness, short of breath, reduced stress, variable speech and imprecise articulation. Furthermore, breathy dysphonia, inappropriate silences and reduced loudness are also speech and vocal patterns evident in hypokinetic dysarthria. Thus, all the symptoms mentioned above were evident and described within the self-reports of the participants. Therefore, to answer R1, we can conclude that the speech and vocal changes are evident via the deviations mentioned above in which it is crystalline that the participants in this study showed positive indicators of severe hypokinetic dysarthria which is causing these speech and vocal changes. 
R2. Are the speech and vocal changes reported significant enough in order to assist a quicker diagnosis in Young-Onset Parkinson's Disease?

Given the answers to R1, where it was highlighted that a vast majority of the participants showed clinical symptoms of severe hypokinetic dysarthria, the speech and vocal changes reported are significant enough to assist a quicker diagnosis for YOPD (Logemaan et al., 1978).

As Hypokinetic Dysarthria occurs in close to $80 \%$ of patients with Parkinson's disease and often may be the primary concern in the early stages of the disease (Ho et al.,1999; Logemann et al., 1978), the changes in speech and voice that occurred in the participants within this study were significant enough in order for a knowledgeable practitioner to be suspicious or recommend that the individual goes through screening for Young Onset Parkinson's Disease.

In addition, living with untreated hypokinetic dysarthria can have devastating effects on daily communication at work, in relationships and with caregivers, causing an increased negative psychosocial impact (Fox \& Ramig, 2017). Thus, earlier detection can also lead to appropriate therapies in order for newly diagnosed patients to come to terms and also learn how to cope with new speech and vocal changes affecting their day-to-day communication. Furthermore, although the motor speech disorder of dysarthria is a primary communication grievance of patients with $\mathrm{PD}$, as the disease progresses other aspects of communication will begin to deteriorate such as lexical retrieval and comprehension of figurative language have been reported as well as with grammaticality, syntactic complexity and information content (Savić, 2017). Furthermore, the presence of hypomimia will begin to manifest, a reduction in facial expression, and thus, also limiting the patient's communication needs, and overall quality of life (Gunnery et al., 2016). 
Thus, if suspicion of hypokinetic dysarthria is detected early on, patients with YOPD can receive early intervention and treatment and thus, extend the delay of the manifestation of these symptoms.

R3. What is the impact on the participants of the study as a consequence of self-reported speech and vocal changes by Young-Onset Parkinson's Patients?

A clear impact from the survey is seen to affect the psycho-social domain, as $88.5 \%$ of the study reported emotional and social distress that affected their day-to-day life, which was instigated because of the speech and vocal changes. The term "psycho-social" is multi-dimensional and defined as "the psychological and social consequences of a motor speech disorder with quality of life, subjective well-being, and societal participation, viewed predominantly as consequence or factors that contribute to psychosocial impact" (Walshe, 2010, p. 97). The participants reported reports of isolation and embarrassment of the changes that had occurred. These emotions are bound to impact one's identity. The speech and voice of one's person mark their identity, personality and how we transmit ourselves to others via communication. Thus, a sudden change in speech and voice can lead to feelings of shame, fearfulness, exhaustion, isolation and embarrassment, among others, as one struggles to navigate themselves with these changes. The psycho-social symptoms expressed by the participants as accumulating due to the impact of speech and vocal change is of great concern; we see negative psychological maladjustment, which is linked to higher rates of depression as a consequence of negative attributional style (Tangney et al., 1992). 
Furthermore, negative psychological maladjustment is also linked to social anxiety (Greene et al., 2003). Additionally, a handful of the participants also expressed that their speech and vocal changes affected their work status as they could no longer continue the work they had previously done and also experienced relationship issues with their significant others. However, dispute clear indications that speech and voice was affecting the psycho-social domains of the participants $79.1 \%$ of the participants were never referred to a speech and language therapist. Additionally, in the sample of this study those who suffered from speech and vocal changes, $31.4 \%$ reported that Levodopa helped alleviate the speech and vocal changes. Thus, it is clear that despite the impact of speech and language, the medical practitioners involved in the cases of the participants of these studies failed to guide them appropriately in order to receive treatment and aid and suggested speech and language therapy for Parkinson's patients which could have significantly improved their day-to-day activities in the domain of communication.

Additionally, another impact of their social and vocal changes was that clinical practitioners did not take them seriously due to their young age and the general expectation that Parkinson's Disease is a neurodegenerative disorder associated with a geriatric population, and thus, they were stigmatized as being "too young" to qualify for Parkinson's Disease. This led to added emotional distress, in which many participants took a long time to be diagnosed with Parkinson's Disease. Furthermore, they felt that they had lost time in their diagnosis which during this time frame, their symptoms became increasingly worse. Finally, many of the participants reported receiving a late diagnosis, or even a misdiagnosis, due to the practitioner's lack of awareness of YOPD, and the preconceived idea that Parkinson's Disease is only seen in a 
geriatric population. Thus, the stigma of neurodegenerative diseases within a younger population must be highlighted in clinical practice and neuroscience research.

To sum up, the speech and vocal changes experienced by the participants in this study YOPD are significant enough to impact them in the psycho-social domain. Therefore, attention should be paid to an increased vulnerability to deteriorating mental health; it must be recognized amongst researchers, clinicians, patients, and caregivers. This is increasingly important as PD is associated with a significant impact on the quality of life of patients, which is dependent on factors such as disease severity and disability, as well as neuropsychiatric symptoms like depression as evident with this sample $6.9 \%$ of participants initial symptom was depression whilst $10.3 \%$ of participants initial symptom was anxiety. Additionally, there is scarce information on the psycho-social impact of Parkinson's disease on those with YOPD, which consequently are rarely addressed in clinical practice nor studied in neuroscience research. However, the available research indicates that age-onset directly impacts psycho-social domains in which their prognosis is worse in younger patients than in older patients. This may be due to being young and mobile and quickly gaining a disability associated with the geriatric population. Furthermore, being young and seeing the alternations in one's body due to the motor and non-motor issues and hallucinations, which may be caused by taking levodopa for many years from a young age, may make the young-onset population more vulnerable. Thus, the answer to $\mathbf{R 2}$ highlights an essential avenue for further exploration in research. 


\section{Proposals for Future Research and Clinicians}

Overall, future researchers must focus on the subgroup of YOPD amongst patients with PD and compare the onset of vocal and speech changes to those who have late-onset Parkinson's disease. Thus, a proposal on more neurolinguistic studies to be conducted YOPD is necessary in order to get a more complete understanding on YOPD. At the moment, there is scarce literature and studies on patients with YOPD, and as a group, they are often overshadowed; thus, more studies must be conducted to understand, treat and support these patients just as much as those with late-onset Parkinson's disease. Furthermore, speech treatments tailored for a younger population with a lifetime of the disease compared to those with late-onset Parkinson's disease should be re-examined and reassessed. Finally, more longitudinal studies that focus on the psycho-social impact of YOPD in patients should remain a focus, especially to explore YOPD concerning self-identity, perception, and astigmatism. Such evidence is urgently needed in order to understand patients with YOPD better and for medical practitioners to be able to recommend better multidisciplinary treatment options.

This study wants to highlight an overall proposal and message for clinical practitioners to become more knowledgeable on YOPD and not pre-judge their patients based on their age. Additionally in terms of clinical management; conferences, medical schools and curriculums in Neurology and Neuroscience should push to educate their doctors in subgroups like YOPD that may go under the radar by clinical practitioners. Given the current medical technology and knowledge we have, there should not be patients who are not heard and subsequently receiving 
missed or late diagnosis, which for neurodegenerative diseases, time can be costly. As mentioned by a participant of this study, "you are never too young to be diagnosed."

\section{Limitations}

Although this study accounted for variables, this study is not without susceptibility towards biases:

1. This study is susceptible to research bias, as open questions within the questionnaire are open to the researcher's interpretation.

2. This study is also susceptible to response bias, as participants may not provide accurate or honest answers, especially in an online survey where confirmation of the disorder has not been done via medical papers provided to the participants by their medical practitioners.

3. This study is susceptible to language bias, as the community groups that were approached were English speakers. This is due to the linguistic restrictions of the researcher. 


\section{Conclusion}

YOPD is a unique subgroup among patients with PD. This study highlighted that, despite the young age of the participants, the majority of them suffered from speech and vocal changes, which resembled the clinical profile of severe hypokinetic dysarthria. The motor speech disorder of dysarthria can cause devastating effects on communication in individuals with PD, and this was evident within the sample of this study, in which the changes in speech and voice affected the psycho-social domains of these participants. Thus, it is concluded that the participants' speech and vocal changes reported in this study are significant. Thus, this is important for

clinical settings as important details on speech and vocal changes can aid medical practitioners to isolate a YOPD case and thus, avoid a late misdiagnosis. However, it is also vital that medical practitioners are aware of this subgroup of PD to be salient to the disorder when they are giving diagnostic assessments to potential PD patients. Whilst this study highlighted the devastating effects on speech and voice that YOPD may have, the results of this study are essential to break stigmas and open the conversation in neuroscience and neurology on YOPD. This study can lead to future contributions from other researchers to better understand how details on speech and voice can aid in the early detection of Parkinson's disease and fine-tune effective treatment strategies tailored for improving intelligibility, social participation, and quality of life with those that have YOPD. 


\section{Acknowledgements}

For this research to become a reality I would like to express my sincere gratitude to my research supervisor Dr. Nerea Ortega Castro for her invaluable support and insight during the writing process of this research. I would also like to thank SAERA (School of Advanced Education, Research and Accreditation S.L), Spain, for encouraging my academic growth. Additionally, I would like to thank all the participants who took part in this research and personally thank the Young Onset Parkinson's Community, who trusted me and shared their journey and personal stories. You showed me that being a researcher also means not forgetting the human element of our work. Finally, I would like to dedicate this research to Theodore Delaportas, my grandfather, who, like many others also suffered from Parkinson's Disease, he never gave up on himself and showed me and those around him the true meaning of strength, survival and courage.

\section{Conflict of Interest}

No economic interest or any kind of other conflict of interest exists. All data generated or analysed during this study are included in this published article and its supplementary information files. 


\section{Reference List}

Anwar, A., Saleem, S., Akhtar, A., Ashraf, S., \& Ahmed, M. F. (2019). Juvenile Parkinson disease. Cureus. Retrieved from https://doi.org/10.7759/cureus.5409

Brabenec, L., Mekyska, J., Galaz, Z., and Rektorova, I. (2017). Speech Disorders in Parkinson's disease: early diagnostics and effects on medication in brain stimulation. J. Neural Transm. 124, 303-334. doi: 10.1007/s00702-017-1676-0

Ciucci, M. R., \& Hoffman, M. (2011). Faculty opinions recommendation of quantitative acoustic measurements for characterization of speech and voice disorders in early untreated Parkinson's disease. Faculty Opinions - Post-Publication Peer Review of Biomedical Literature. Retrieved from https://doi.org/10.3410/f.8677957.9190055

De Lau, L. M., Koudstaal, P. J., Hofman, A., \& Breteler, M. M. (2006). Serum cholesterol levels and the risk of Parkinson's disease. American Journal of Epidemiology, 164(10), 998-1002. Retrieved from https://doi.org/10.1093/aje/kwj283

Duffy, J. R. (2012). Motor speech disorders: Substrates, differential diagnosis, and management. Elsevier Health Sciences.

Darley, F. L., Aronson, A. E., \& Brown, J. R. (1969). Clusters of deviant speech dimensions in the dysarthrias. Journal of Speech and Hearing Research, 12(3), 462-496. https://doi.org/10.1044/jshr.1203.462 
Grant, L., Rajamanickam, E., Hilby, B., Blue, K., Jones, C., Kelm-Nelson, C., \& Ciucci, M. (2013). Early identification and treatment of communication and swallowing deficits in Parkinson disease. Seminars in Speech and Language, 34(03), 185-202. Retrieved from https://doi.org/10.1055/s-0033-1358367

Green, S., Moll, J., Deakin, J. F., Hulleman, J., \& Zahn, R. (2013). Proneness to decreased negative emotions in major depressive disorder when blaming others rather than oneself. Psychopathology, 46(1), 34-44. https://doi.org/10.1159/000338632

Gunnery, S. D., Habermann, B., Saint-Hilaire, M., Thomas, C. A., \& Tickle-Degnen, L. (2016). The relationship between the experience of Hypomimia and social wellbeing in people with Parkinson's disease and their care partners. Journal of Parkinson's Disease, 6(3), 625-630. https://doi.org/10.3233/jpd-160782

Ho, A. K., Iansek, R., Marigliani, C., Bradshaw, J. L., \& Gates, S. (1999). Speech impairment in a large sample of patients with Parkinson's disease. Behavioral Neurology, 11(3), 131-137. Retrieved from https://doi.org/10.1155/1999/327643

Fénelon, G., Mahieux, F., Huon, R., \& Ziégler, M. (2000). Hallucinations in Parkinson's disease: Prevalence, phenomenology and risk factors. Brain, 123, 733-745.

Fox, C. M., \& Ramig, L. O. (1997). Vocal sound pressure level and self-perception of speech and voice in men and women with idiopathic Parkinson disease. American Journal of Speech-Language Pathology, 6(2), 85-94. https://doi.org/10.1044/1058-0360.0602.85 
Ho, A. K., Iansek, R., Marigliani, C., Bradshaw, J. L., \& Gates, S. (1999). Speech impairment in a large sample of patients with Parkinson's disease. Behavioural Neurology, 11(3), 131-137. Retrieved from https://doi.org/10.1155/1999/327643

Kegl J., Cohen H., Poizner H. (1999) Articulatory consequences of Parkinson's Disease: perspectives from two modalities. Brain Cogn 40:355-386

Logemann, J. A., Fisher, H. B., Boshes, B., \& Blonsky, E. R. (1978). Frequency and Co Occurrence of vocal tract dysfunctions in the speech of a large sample of Parkinson patients. Journal of Speech and Hearing Disorders, 43(1), 47-57. Retrieved from https://doi.org/10.1044/jshd.4301.47

Mekyska, J., Smekal, Z., Galaz, Z., Mzourek, Z., Rektorova, I., Faundez-Zanuy, M., \& López-de-Ipiña, K. (2016). Perceptual features as markers of Parkinson's disease: The issue of clinical Interpretability. Recent Advances in Nonlinear Speech Processing, 83-91. Retrieved from https://doi.org/10.1007/978-3-319-28109-4_9

Maffoni, M., Giardini, A., Pierobon, A., Ferrazzoli, D., \& Frazzitta, G. (2017). Stigma experienced by Parkinson's disease patients: A descriptive review of qualitative studies. Parkinson's Disease, 2017, 1-7. Retrieved from https://doi.org/10.1155/2017/7203259

Przedborski, S., Vila, M., \& Jackson-Lewis, V. (2003). Neurodegeneration: what is it and where are we?. The Journal of clinical investigation, 111(1), 3-10. Retrieved from https://doi.org/10.1172/JCI17522 
Poluha, P.C., Teulings, H.L., Brookshire, R.H (1998). Handwriting and speech changes across the levodopa cycle in Parkinson's disease. Acta Psychol. 1 100, 71-84

Parkinson, J. (2002). An essay on the shaking palsy. J. Neuropsychiatry Clin. Neurosci. 14, 223-236. Retrieved from https://doi.org/10.1176/jnp.14.2.223

Thompson AWS: ON being a parkinsonnian. In Kapur, N. (1997). Injured brains of medical minds: Views from within. Oxford University Press.

Skodda S. (2012) Effect of deep brain stimulation on speech performance in Parkinson's disease. Parkinson's Disease. Retrieved from 10. 1155/2012/850596

Sung, H. Y., Kim, J., Lee, K., Kim, Y., Song, I., Chung, S., Yang, D., Cho, Y. K., Park, J. M., Lee, I. S., Kim, S. W., Chung, I., \& Choi, M. (2010). The prevalence and patterns of pharyngoesophageal dysmotility in patients with early stage Parkinson's disease. Movement Disorders, 25(14), 2361-2368. Retrieved from https://doi.org/10.1002/mds.23290

Špica, V., Pekmezović, T., Svetel, M., \& Kostić, V. S. (2012). Prevalence of non-motor symptoms in young-onset versus late-onset Parkinson's disease. Journal of Neurology, 260(1), 131-137. Retrieved from https://doi.org/10.1007/s00415-012-6600-9

Savić, G. (2017). Speech impairment, phonation, writing, salivation, and swallowing in patients with Parkinson's disease. Advances in Speech-language Pathology. https://doi.org/10.5772/intechopen.69711 
Serván, B. (2018). Non-motor symptoms in Parkinson disease: Impulse-control disorder. A case report. Retrieved from https://doi.org/10.26226/morressier.5a6ef3eed462b80290b5797c

Tangney, J. P., Wagner, P., \& Gramzow, R. (1992). Proneness to shame, proneness to guilt, and psychopathology. Journal of Abnormal Psychology, 101(3), 469-478. https://doi.org/10.1037/0021-843x.101.3.469

Volont, M., Porta, M., \& Comi, G. (2002). Clinical assessment of dysphagia in early phases of Parkinson's disease. Neurological Sciences, 23(0), s121-s122. Retrieved from https://doi.org/10.1007/s100720200099

Walshe M., (2010). The psychosocial impact of acquired motor speech disorders. In: Lowit A, Kent RD, editors. Assessment of Motor Speech Disorders. 97-122.

Yunusova, Y., Weismer, G. G., Westbury, J. R., and Lindstrom, M. J. (2008). Articulatory movements during vowels in speakers with dysarthria and healthy controls. J. Speech Lang. Hear. Res. 51, 596-611. Retrieved from https//doi.org/10.1044/1092-4388 


\section{Appendices}

\section{Appendix A}

A copy of the informed consent that was signed on google forms before the participants were included in the study.

This questionnaire is part of a research project conducted at SAERA (Advanced Education, Research and Accreditation) named "Detection of Parkinson's Disease through Speech and Vocal Changes in Young Onset Patients: A Survey" in the context of my MA Thesis for the course in Neuroscience (Specialising in Neurological Speech Therapy). All response data is anonymous. No personal details will be collected. You have the right to refuse to participate or withdraw at any time without any penalty. If you submit the survey, I have the right to use the information provided for my research in regards to your experience with young onset Parkinson Disease. The questionnaire has open and closed ended questionnaires regarding your personal experience with early speech and vocal changes in young onset Parkinsons Disease. This questionnaire should take you no more than 10 minutes to complete. If someone else is typing your answers, you give them permission to submit the information on behalf of yourself. Please indicate that you agree with the informed consent and that you accept for your information to be used for the context of this research project. *

I agree with the statement made above and want to take part in this study. 